\title{
NSF Graduate-Level Research Facilities Program
}

The National Science Foundation has announced that December 1, 1962, will be the next closing date for receipt of proposals by its Office of Institutional Programs for the renovation or construction of graduate-level research facilities. This will be followed by closing dates of April 1 and August 1, 1963. Proposals may be submitted by departments having at least a master's program in the life, social, mathematical, engineering, or physical sciences. Funds are granted on a 50\% matching basis. During FY 1962, 224 grants were awarded for a total of $\$ 26.1$ million. A booklet describing the Graduate-Level Research Facilities Program may be obtained from the National Science Foundation, Washington 25, D. C.

Clyde C. Hall Public Information Officer

\section{NEW BOOKS RECEIVED}

The Astronomical Universe, 2nd ed., by Wasley S. Krogdahl. Published by the MacMillan Company, New York. 585 pages. Price $\$ 7.95$.

Second, revised edition of one of the better-written introductory texts. Much improved illustrations; outstanding chapter on cosmology.

Astronomischer Jahresbericht, by W. Lohmann, F. Henn. Published by Walter de Gruyter and Company, Berlin, 1962.

Summary index to the astronomical literature of 1960.

Researches on Meteorites, edited by Carleton B. Moore. Published by John Wiley \& Sons, Inc., New York, 1962. 227 pages. Price $\$ 7.00$.

Heterogeneous collection of papers, mostly available elsewhere as well, touching various aspects of meteorites.

The Shift and Shape of Spectral Lines, by Robert G. Breene, Jr. Published by Pergamon Press Ltd., Oxford, London, New York, 1961. 236 pages. Price $\$ 15.00$.

A theoretical treatment quite specifically of the subjects indicated by the title. It leans heavily upon the original papers in the field and includes an extensive bibliography. Good introductory historical chapter on line-broadening theory; the remainder recommended as a reference work for specialists.

Fundamentals of Celestial Mechanics, by J. M. A. Danby. Published by the Macmillan Company, New York, 1961. 348 pages. Price $\$ 8.00$.

Long-needed complete and accurate development of basic celestial mechanics from first principles, organized for teaching as a full-year course at advanced undergraduate or introductory graduate level. Many problems.

Proceedings of the Fourth Symposium on Cosmical Gas Dynamics, Aerodynamic Phenomena in Stellar Atmospheres. Published as International Astronomical Union Symposium No. 12, by Nicola Zanichelli, Bologna.

Book-length proceedings of the August 1960 symposium held at Lake Como. Original papers and high-level discussions are reported in detail. Principal topics covered: Questions of general background and methodology relating to aerodynamic phenomena in stellar atmospheres; General summary of results on "astronomical turbulence" in stellar atmospheres; Spherically symmetric motions in stellar atmospheres (A) Pulsating variable stars, (B) The propagation of a shock wave in an atmosphere of varying density, (C) Noncatastrophic mass loss from stars; Considerations on localized velocity fields in stellar atmospheres: Prototype-the solar atmospheres, (A) Convection and granulation: preview on granulation-observational studies, (B) Considerations of convective instability from the viewpoint of physics, (C) Transient velocity fields in the lower solar atmosphere, (D) Collision-free shock waves, and Summaries.

Manual for Astronomical Photoelectric Photometry, by the Photoelectric Photometry Committee of the American Association of Variable Star Observers. Published by the AAVSO, Cambridge, Massachusetts, 1962. 21 pages. Price $\$ 1.00$.

Useful little pamphlet for those contemplating photoelectric photometry. Summarizes the most basic references, techniques, circuits and suppliers. Mainly intended for amateurs. 


\section{ERRATA}

Astron. J. 65, 448 .

Last line, left column, for $m_{p v}$ read $M_{p v}$.

Astron. J. 66, 50.

Fourth line of text, for $\gtrsim 3 \%$ read $\leqslant 3 \%$.

Astron. J. 66, 516.

For expi $\chi=\left(1-\eta_{0}^{2} \sin \varphi\right)$ read expi $\chi=\left(1-\eta_{0}^{2} \sin ^{2} \varphi\right)$.

Astron. J. 67, No. 1.

Back cover page, article by Binnendijk for ER Leonis read ER Orınis.

Astron. J. 67, 177.

Equation for $\xi$, for $\left(1-e^{2}\right)^{3 / 2} \operatorname{read}\left(1-e^{2}\right)^{-3 / 2}$.

Astron. J. 67, 163.

In the two summation equations for $k$ which precede Table I, for "cos" read "cosec."

In the second paragraph following Table I, in the definition for $m_{2}$ for " $\rho$ " read " $\mu$ " 